\title{
(6) OPEN ACCESS \\ How close are we to standardised extended RAS gene mutation testing? The UK NEQAS evaluation
}

\author{
Susan D Richman, ${ }^{1}$ Jennifer Fairley, ${ }^{2}$ Rachel Butler, ${ }^{3}$ Zandra C Deans ${ }^{2}$
}

\begin{abstract}
${ }^{1}$ Department of Pathology and Tumour Biology, Leeds Institute of Cancer and Pathology, St James University Hospital, Leeds, UK

${ }^{2}$ UK NEQAS for Molecular Genetics, Department of Laboratory Medicine, The Royal Infirmary, Edinburgh, UK ${ }^{3}$ Cardiff and Vale UHB-Medical Genetics University Hospital of Wales, Cardiff, Wales, UK
\end{abstract}

\section{Correspondence to} Dr Zandra C Deans, UK NEQAS for Molecular Genetics, Department of Laboratory Medicine, The Royal Infirmary, Edinburgh EH16 4SA, UK; Sandi.Deans@ed.ac.uk

Received 20 April 2016 Revised 19 May 2016 Accepted 20 May 2016 Published Online First 28 September 2016

\section{CrossMark}

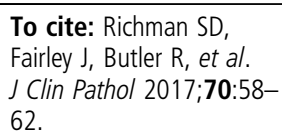

\section{ABSTRACT}

Aims Since 2008, KRAS mutation status in exon 2 has been used to predict response to anti-EGFR therapies. Recent evidence has demonstrated that NRAS status is also predictive of response. Several retrospective 'extended $R A S^{\prime}$ analyses have been performed on clinical trial material. Despite this, are we really moving towards such extended screening practice in reality?

Methods Data were analysed from four consecutive UK National External Quality Assessment Service for Molecular Genetics Colorectal cancer External Quality Assessment schemes (during the period 2014-2016), with up to 110 laboratories (worldwide) participating in each scheme. Testing of four or five tumour samples is required per scheme. Laboratories provided information on which codons were routinely screened, and provided genotyping and interpretation results for each sample. Results At least $85 \%$ of laboratories routinely tested KRAS codons 12,13 and 61. Over the four schemes, an increasing number of laboratories routinely tested KRAS codons 59, 117 and 146. Furthermore, more laboratories were introducing next generation sequencing technologies. The pattern of 'extended testing' was reassuringly similar for NRAS, although fewer laboratories currently test for mutations in this gene. Alarmingly, still only $36.1 \%$ and $24.1 \%$ of participating laboratories met the ACP Molecular Pathology and Diagnostics Group and American Society of Clinical Oncology guidelines, respectively, for extended RAS testing in the latest assessment.

Conclusions Despite recommendations in the UK and USA on extended RAS testing, there has clearly been, based on these results, a delay in implementation. Inadequate testing results in patients being subjected to harmful treatment regimens, which would not be the case, were routine practice altered, in line with evidencebased guidelines.

\section{INTRODUCTION}

Over the past few years, KRAS mutation testing has become compulsory as a prerequisite for the treatment of patients with metastatic colorectal cancer (mCRC), with anti-EGFR monoclonal antibody $(\mathrm{mAb})$ therapies such as cetuximab or panitumumab. It has been well established that patients with mutations in exon 2 (codons 12 and 13) of KRAS fail to respond to anti-EGFR therapy and display inferior outcomes when therapy is combined with oxaliplatin-based chemotherapy. ${ }^{1-5}$ It is also apparent that a significant proportion of exon 2 wildtype (WT) patients gain no benefit from anti-EGFR therapy. This has led to the need for additional refinement of the patient population receiving such therapy, in order to better select patients for treatment, and equally identify those who will gain no benefit from a toxic drug regimen.

Recently available data, resulting primarily from the reanalysis of several large randomised controlled trials (RCTs), have provided evidence for the introduction of an extended RAS testing panel, to include NRAS testing and also to cover significantly more mutation hotspots, in KRAS. A prospective-retrospective analysis was carried out on the phase III PRIME study ${ }^{6}$ to evaluate the treatment effect of panitumumab-FOLFOX4 (Pan-FOLFOX4), when compared with FOLFOX4 alone, in patients with RAS-WT (KRAS and NRAS exons 2, 3 and 4 WT) and patients with RAS-WT plus BRAF-WT (KRAS and NRAS exons 2, 3 and 4 and BRAF exon 15 WT). Both overall survival (OS) and progressionfree survival (PFS) were significantly increased with Pan-FOLFOX4. Furthermore, $17 \%$ of the 639 patients with mCRC, with no KRAS mutation in exon 2, had additional RAS mutations, which were associated with worse PFS and OS, when treated with Pan-FOLFOX4.

Last year, data were published from the phase III CRYSTAL trial ${ }^{7}$ where extended RAS testing was carried out on the mCRC KRAS exon 2 WT trial population. As was seen in the PRIME study, both OS and PFS were significantly increased in the patients receiving cetuximab plus FOLFIRI, compared with those receiving FOLFIRI alone. The percentage of patients who carried RAS mutations in addition to KRAS exon 2 was $14.7 \%$, which is similar to the level identified in the patients in the PRIME trial (17\%).

The data from these two large trials, in combination with the data from a further 13 articles, ${ }^{8-19}$ formed the basis of the recent American Society of Clinical Oncology (ASCO) Provisional Clinical Opinion (PCO) update. ${ }^{20}$ The recommendation of the PCO was that KRAS exon 2 (codons 12 and 13) mutations should be assessed in addition to KRAS exon 3 (codons 59 and 61) and exon 4 (codons 117 and 146), along with NRAS exon 2 (codons 12 and 13), exon 3 (codons 59 and 61) and exon 4 (codons 117 and 146).

Current European guidelines for prescribing anti-EGFR monoclonal antibody therapy (http:// www.ema.europa.eu/docs/en_GB/document_library/ Summary_of_opinion/human/000741/WC5001448 27.pdf) state that 'Vectibix (Panitumumab) is indicated for the treatment of adult patients with wildtype RAS metastatic colorectal cancer (mCRC)', without specifically stating which codons must be tested. In the UK, however, the Association of Clinical Pathologists Molecular Pathology and Diagnostics Group devised a set of guidelines, directed at UK practice and more specifically within the 
National Health Service (NHS). ${ }^{21}$ The recommendation was that RAS testing for treatment with anti-EGFR therapies should include, as a minimum, KRAS codons 12, 13, 59, 61, 117 and 146 and NRAS codons 12, 13,59 and 61. As these codons account for over $99 \%$ of $R A S$-activating mutations, ${ }^{22}$ UK National External Quality Assessment Service (UK NEQAS) for Molecular Genetics has provided assessment of molecular testing of colorectal cancer since $2008^{23}$ and has determined that it is good practice for all of these to be covered by laboratories participating in their External Quality Assessment (EQA) schemes.

Given that there is now adequate data available confirming the need to perform extended RAS testing, we report here on the very varied range of $R A S$ (KRAS and NRAS) codons being routinely tested for mutation detection, across four UK NEQAS for Molecular Genetics Colorectal cancer EQA schemes, with laboratory testing being performed between October 2014 and November 2015, in up to 110 UK and international laboratories.

\section{MATERIALS AND METHODS}

The UK NEQAS for Molecular Genetics EQA scheme for the molecular genetic analysis of colorectal cancer is provided twice during each 12-month period, with approximately 6 months between each run. Multiple formalin-fixed, paraffin-embedded (FFPE) colorectal tumour tissue blocks were sourced to include a number of WT and RAS mutations, and variable sample processing/fixation processes into the scheme. To ensure the samples distributed for the EQA scheme gave reportable accurate $R A S$ results, each sample was tested in two independent, validating laboratories using two different testing methods, prior to being sent out to participating laboratories. Consent was in place to allow the use of this excess pathological material in the UK NEQAS EQA scheme. Data were available from the two runs delivered in 2014-2015 (Runs 1 and 2) and the two runs delivered during 2015-2016 (Runs 1 and 2). Each year, participating laboratories are sent samples with five fictitious clinical scenarios in Run 1 and four in Run 2. Laboratories are expected to test these nine samples in accordance with local laboratory practice and report results for both KRAS and NRAS testing. It was deemed optional as to whether laboratories chose to report $B R A F$ and PIK3CA testing results. Each laboratory was required to stipulate the preferred sample type: rolled sections for each case; rolled sections plus a slide-mounted section or only slidemounted sections, and all requests were met by UK NEQAS. An estimate of tumour percentage was expected from each laboratory, for each tumour sample, or a statement indicating that tumour assessment was not carried out. None of the tumour samples distributed had less than $20 \%$ tumour cell content. Each laboratory was also required to provide a clinical interpretation of their results according to the clinical cases provided, and state the methodologies used for mutation detection.

\section{RESULTS}

\section{Scheme participation}

Eighty-six laboratories registered to participate in Run 1 of the 2014-2015 scheme, and of these, 84 submitted results. Ninety-one laboratories registered to participate in Run 2, and again 84 submitted results. In the 2015-2016 scheme, 101 laboratories registered to participate in Run 1, and 99 submitted results, and 108/120 registered laboratories returned results in Run 2. The two laboratories in Run 1 and six laboratories in Run 2 failing to submit reports in the 2014-2015 scheme followed the correct protocol, and contacted the Scheme to advise them of the non-submission of results. Likewise, the two
Table 1 Distribution of sample types requested by the laboratories, registering for each of the four schemes

\begin{tabular}{lllll}
\hline & $\begin{array}{l}\text { Run 1 } \\
\mathbf{2 0 1 4 - 1 5} \\
(\mathbf{n = 8 6 )}\end{array}$ & $\begin{array}{l}\text { Run 2 } \\
\mathbf{2 0 1 4 - 1 5} \\
(\mathbf{n = 9 1 )}\end{array}$ & $\begin{array}{l}\text { Run 1 } \\
\mathbf{2 0 1 5 - 1 6} \\
(\mathbf{n}=101)\end{array}$ & $\begin{array}{l}\text { Run 2 } \\
\mathbf{2 0 1 5 - 1 6} \\
(\mathbf{n}=120)\end{array}$ \\
\hline $\begin{array}{l}\text { Mounted FFPE } \\
\text { section only }\end{array}$ & $34(40 \%)$ & $34(37 \%)$ & $37(37 \%)$ & $46(38 \%)$ \\
$\begin{array}{l}\text { Rolled section plus } \\
\text { mounted FFPE }\end{array}$ & $36(42 \%)$ & $40(44 \%)$ & $46(46 \%)$ & $53(44 \%)$ \\
$\begin{array}{l}\text { section } \\
\text { Rolled section only }\end{array}$ & $16(19 \%)$ & $17(19 \%)$ & $18(18 \%)$ & $21(18 \%)$ \\
\hline
\end{tabular}

For each run, not every laboratory requesting material went on to complete their submission

FFPE, formalin-fixed, paraffin-embedded.

laboratories in Run 1 and seven laboratories in Run 2 failing to report results for the 2015-2016 scheme also followed the correct protocol. However, one laboratory in Run 2 of the 2014-2015 scheme and five laboratories in Run 2 of the 20152016 scheme were deemed poor performing laboratories due to non-submission in accordance with the published guidelines.

\section{Sample type tested}

The distribution of sample types requested did not vary much across the four schemes. Table 1 shows the percentage of laboratories requesting mounted FFPE tumour sections only, rolled sections of tissue plus a slide-mounted section or rolled sections only.

\section{Regions of KRAS and NRAS tested}

A comparison of the regions of KRAS and NRAS covered by mutation screening across the three schemes was performed. For $K R A S$, there was an increase in the number of laboratories testing each codon, with the largest increases seen in codons 59 , 117 and 146. There was also an increase in the number of laboratories stating just the exons tested (exons 2, 3 and 4), indicative of an increase in the number of laboratories moving to a next generation sequencing (NGS) platform (table 2). For NRAS, a very similar pattern was seen across all codons; however, clearly, there are still fewer laboratories testing NRAS, although a smaller proportion of participating laboratories is failing to report results for NRAS (table 3).

Table 2 Percentage of laboratories in each scheme run, stating the codons (or exons) that were covered in their screening panels for KRAS

\begin{tabular}{lllll}
\hline $\begin{array}{l}\text { Codons or } \\
\text { exons tested }\end{array}$ & $\begin{array}{l}\text { KRAS Run 1 } \\
(\mathbf{2 0 1 4 - 1 5 )} \\
\mathbf{n = 8 4}\end{array}$ & $\begin{array}{l}\text { KRAS Run 2 } \\
(\mathbf{2 0 1 4 - 1 5 )} \\
\mathbf{n}=84\end{array}$ & $\begin{array}{l}\text { KRAS Run 1 } \\
(\mathbf{2 0 1 5 - 1 6 )} \\
\mathbf{n}=99\end{array}$ & $\begin{array}{l}\text { KRAS Run 2 } \\
(\mathbf{2 0 1 5 - 1 6 )} \\
\mathbf{n}=108\end{array}$ \\
\hline Codons 12/13 & 82.1 & 89.3 & 81.8 & 76.9 \\
Codon 59 & 14.3 & 15.5 & 20.2 & 29.6 \\
Codon 61 & 73.8 & 85.5 & 71.7 & 69.4 \\
Codon 117 & 16.7 & 41.6 & 47.5 & 50.9 \\
Codon 146 & 35.7 & 52.4 & 54.5 & 54.6 \\
Exon 2 & 9.5 & 9.5 & 12.1 & 15.7 \\
Exon 3 & 9.5 & 9.5 & 12.1 & 15.7 \\
Exon 4 & 4.8 & 4.8 & 10.1 & 13.9 \\
Not specified & 8.3 & 1.2 & 6.1 & 7.4 \\
\hline
\end{tabular}


Table 3 Percentage of laboratories in each scheme run, stating the codons (or exons) that were covered in their screening panels for NRAS

\begin{tabular}{|c|c|c|c|c|}
\hline $\begin{array}{l}\text { Codons or } \\
\text { exons tested }\end{array}$ & $\begin{array}{l}\text { NRAS Run } \\
1 \text { (2014-15) } \\
\mathrm{n}=84\end{array}$ & $\begin{array}{l}\text { NRAS Run } \\
2 \text { (2014-15) } \\
\mathrm{n}=84\end{array}$ & $\begin{array}{l}\text { NRAS Run } \\
1 \text { (2015-16) } \\
\mathrm{n}=99\end{array}$ & $\begin{array}{l}\text { NRAS Run } 2 \\
(2015-16) \\
\mathrm{n}=108\end{array}$ \\
\hline Codons $12 / 13$ & 58.3 & 73.8 & 68.7 & 66.7 \\
\hline Codon 59 & 14.3 & 22.6 & 23.2 & 28.7 \\
\hline Codon 61 & 71.4 & 73.8 & 65.7 & 63.9 \\
\hline Codon 117 & 15.5 & 20.2 & 21.2 & 21.3 \\
\hline Codon 146 & 21.4 & 26.2 & 30.3 & 29.6 \\
\hline Exon 2 & 10.7 & 10.7 & 13.1 & 15.7 \\
\hline Exon 3 & 10.7 & 10.7 & 13.1 & 15.7 \\
\hline Exon 4 & 6 & 16.7 & 10.1 & 11.1 \\
\hline Not tested & 22.6 & 13.1 & 12.1 & 10.2 \\
\hline Not specified & 8.3 & 1.2 & 6.1 & 7.4 \\
\hline
\end{tabular}

We investigated the gene regions tested in the most recent scheme (Runs 1 and 2 of 2015-2016), which was provided following the updated UK guidelines. ${ }^{21}$ Each laboratory was expected to state which regions of KRAS and NRAS were covered by their screening protocol. Despite this requirement, five laboratories in Run 1 and eight laboratories in Run 2 failed to provide this information on their diagnostic clinical report.

There was a slight decrease in the percentage of laboratories not testing for mutations in NRAS, dropping from 12/99 (12.1\%) to $11 / 108(10.2 \%)$ from Run 1 to Run 2. In Run 1, there were two samples harbouring NRAS mutations: one containing a c.35G $>\mathrm{T}$ and one containing a c.181C $>\mathrm{A}$ mutation. In Run 2, there was one sample harbouring a c.34 G>T mutation. Thus, 12 and 11 laboratories failed to report these mutations in Runs 1 and 2, respectively.

Tables 4 and 5 show the proportion of laboratories meeting, or failing to meet, the UK and US guidelines for extended RAS testing respectively. Despite the increase seen across the two runs, there was still a significant proportion of laboratories that was effectively undertesting.

Taking into account the current UK NEQAS for Molecular Genetics recommendations, only 24/99 (24.2\%) and 39/108 (36.1\%) of laboratories are meeting these minimum requirements in Runs 1 and 2, respectively.

Table 4 The percentages of laboratories either meeting the UK guidelines for extended RAS testing, or indeed overtesting or undertesting

\begin{tabular}{|c|c|c|}
\hline & $\begin{array}{l}\text { Run } 1 \\
(n=99)\end{array}$ & $\begin{array}{l}\text { Run } 2 \\
(n=108)\end{array}$ \\
\hline $\begin{array}{l}\text { Percentage of laboratories exactly following UK } \\
\text { guidelines }\end{array}$ & 6.1 & 25.9 \\
\hline Percentage of laboratories overtesting & 19.2 & 10.2 \\
\hline Percentage of laboratories undertesting & 68.7 & 55.6 \\
\hline $\begin{array}{l}\text { Percentage of laboratories where the testing panel } \\
\text { was undetermined }\end{array}$ & 6 & 8.3 \\
\hline $\begin{array}{l}\text { TOTAL percentage of laboratories testing at least in } \\
\text { accordance with UK guidelines }\end{array}$ & 25.3 & 36.1 \\
\hline
\end{tabular}

Adding together the laboratories following the UK guidelines and those overtesting gives the total percentage of laboratories meeting or exceeding the minimum

requirements. The UK guidelines suggest the following coverage: KRAS codons 12 ,

$13,59,61,117$ and 146 in addition to NRAS codons $12,13,59$ and 61.
Table 5 The percentages of laboratories either meeting the US guidelines for extended RAS testing, or indeed overtesting or undertesting

\begin{tabular}{|c|c|c|}
\hline & $\begin{array}{l}\text { Run } 1 \\
(n=99)\end{array}$ & $\begin{array}{l}\text { Run } 2 \\
(n=108)\end{array}$ \\
\hline $\begin{array}{l}\text { Percentage of laboratories exactly following ASCO } \\
\text { guidelines }\end{array}$ & 4 & 13 \\
\hline Percentage of laboratories overtesting & 15.2 & 11.1 \\
\hline Percentage of laboratories undertesting & 74.8 & 67.6 \\
\hline $\begin{array}{l}\text { Percentage of laboratories where the testing panel } \\
\text { was undetermined }\end{array}$ & 6 & 8.3 \\
\hline $\begin{array}{l}\text { TOTAL percentage of laboratories testing at least in } \\
\text { accordance with ASCO guidelines }\end{array}$ & 19.6 & 24.1 \\
\hline \multicolumn{3}{|c|}{$\begin{array}{l}\text { Adding together the laboratories following the US guidelines and those overtesting } \\
\text { gives the total percentage of laboratories meeting or exceeding the minimum } \\
\text { requirements. The current American Society of Clinical Oncology (ASCO) guidelines } \\
\text { state that the coverage should include KRAS exon } 2 \text { (codons } 12 \text { and 13), KRAS exon } \\
3 \text { (codons } 59 \text { and 61), KRAS exon } 4 \text { (codons } 117 \text { and 146), in addition to NRAS exon } \\
2 \text { (codons } 12 \text { and 13), NRAS exon } 3 \text { (codons } 59 \text { and 61) and NRAS exon } 4 \text { (codons } \\
117 \text { and 146). }\end{array}$} \\
\hline
\end{tabular}

When the current ASCO guidelines are taken into account, only $19 / 99(19.2 \%)$ of laboratories met the recommendations in Run 1, with this rising to 26/108 (24.1\%) in Run 2.

\section{DISCUSSION}

In an era of personalised medicine, it is becoming increasingly important to identify which patients will respond to specific drugs, and equally, which will gain no benefit. Furthermore, additional stratification is required to ensure the greatest benefits are obtained, once patients are selected for individual treatment. It was established in 2008 that KRAS mutation was a negative predictive biomarker of response to panitumumab ${ }^{3}$ and cetuximab. ${ }^{124}$ In these studies, only KRAS codons 12 and 13 were tested for the presence of pathogenic mutations. The PICCOLO trial, run across the UK, was one of the first mCRC randomised trials to introduce prospective mutation testing, allowing randomisation based on mutation status. ${ }^{25}$ Again, only KRAS status, at codons 12, 13 and 61, was assessed prospectively. Based on the evidence of the low response rate to anti-EGFR therapies, the group also carried out a retrospective analysis of additional mutation hotspots (BRAF codon 600; NRAS codons 12, 13 and 61; KRAS codon 146; PIK3CA codons 542, 545-6 and 1047). Patients who were WT at all loci ('all wild-type') demonstrated a high response rate to panitumumab (70/160 (44\%)) and also an improved PFS (HR 0.68; 95\% CI 0.53 to 0.86), whereas patients whose tumours contained any mutation demonstrated a detrimental effect from panitumumab, in terms of PFS (HR 1.20 ; $95 \%$ CI 0.83 to 1.74 ). During 2015 , there was a publication of the extended $R A S$ testing retrospectively carried out on patients in the OPUS trial. ${ }^{26}$ Only 26\% of the 118 evaluable patients were found to harbour an additional RAS mutation. Patients with WT tumours demonstrated an improved objective response with the addition of cetuximab to FOLFOX4 (58\% vs $29 \%$; OR $3.3,95 \%$ CI 1.36 to $8.7 ; \mathrm{p}=0.0084)$, whereas those with any mutation derived no benefit, and indeed demonstrated a detrimental effect. These data, in combination with the extended RAS testing in CRYSTAL and PRIME, have highlighted the need for extended mutation testing, particularly where there is the potential of a detrimental effect on the patient.

Laboratories proving a clinical service in terms of $R A S$ mutation screening should be participating in a regular EQA scheme, to ensure adequate quality measures are met. The UK NEQAS 
for Molecular Genetics Colorectal cancer EQA scheme has observed an increase in the number of laboratories participating and in the breadth of codons screened by participants over the course of the past four EQA runs (October 2014 to November 2015). It was noted that not all laboratories carry out a pathology review of each tumour sample. Almost $20 \%$ of participating laboratories requested rolled sections only for testing, and were thus unable to assess the tumour content, as requested in the scheme. These laboratories were not penalised, providing an indication was given on the laboratory report, that this was the case. UK NEQAS would rather assess each laboratory's routine process; so, if they do not routinely assess tumour content, yet report clinical results, it is preferable that this is assessed by the EQA provider, rather than routine processing be amended for scheme participation. Most markedly have been increases in the number of laboratories now including KRAS codons 59, 117 and 146 in their screening panels. Furthermore, there has been a substantial increase in the number of laboratories including NRAS testing into the testing strategies. Increased implementation of NGS panel testing was seen across the four runs examined. In Run 1 of 2014-2015, only $9.5 \%$ of laboratories were using NGS to screen for mutations on KRAS, and $11.9 \%$ were using NGS to screen NRAS. This increased to $23.1 \%$ in Run 2 of 2015-2016, for both KRAS and NRAS. It was observed that the format of reporting NGS results was highly variable, and many reports did not state the gene panel or sequencing platform used. As this is not a scheme requirement, laboratories were not penalised for failing to provide this information. The implementation of gene panel testing is clearly becoming more routine practice, as testing requirements increase. This will become more challenging as the demand for large-panel gene mutation screening on small, diagnostic biopsies, where the limiting factor will be the tissue sample itself, increases.

As previously mentioned, in both USA and UK, there are now published guidelines on extended RAS testing. ${ }^{20}{ }^{21}$ Alarmingly, we have shown here that of the laboratories $(n=108)$ participating in the most recent EQA scheme, only $24.1 \%$ of laboratories met the ASCO guidelines and $36.1 \%$ met the UK guidelines. Figures based on the CRYSTAL and PRIME ${ }^{7} 7$ studies would suggest that although $40 \%$ of patients with mCRC are likely to have a KRAS exon 2 mutation, there are still between $14.7 \%$ and $17 \%$ of patients with an additional $R A S$ mutation, providing strong evidence for extended RAS testing. In the USA, there are currently two FDA-approved mutation screening kits (Therascreen RGD PCR kit and the Roche cobas KRAS mutation testing kit), both of which only cover seen mutations in codons 12 and 13 of KRAS. These kits are clearly a very long way from meeting the ASCO guidelines, yet may have to be used in certain laboratories. In Europe, the European Medicines Agency (EMA) is not prescriptive as to the testing methodologies or scope of testing, which enables laboratories to introduce in-house developed tests or other commercially available kits, which incorporate wider RAS testing. Each laboratory therefore has the choice of determining the extent of testing in accordance with best-practice guidance. However, the implementation of new tests requires validation and verification, which are costly, and many testing centres have no resources for such validation. This may go some way to explaining the limited and different testing strategies employed across the scheme participants. Clearly, there is still a long way to go before these guidelines are followed in routine practice, and until this happens, we will see a larger than necessary patient cohort, subjected to the detrimental effects of their anti-EGFR therapies, which would not have been prescribed, had an extended $R A S$ panel been incorporated into practice.

\section{Take home messages}

- We have provided evidence of the nature of current RAS testing, across 100 global laboratories, in the context of an international quality assurance scheme.

- We have demonstrated the alarming lack of full extended RAS testing, in accordance to both UK and US guidelines.

- Practice must be altered to bring laboratories in line with these evidence-based guidelines to ultimately provide a high standard of patient care.

\section{Handling editor Runjan Chetty}

Contributors All authors were involved in the analysis and interpretation of data, writing the manuscript and in the decision to submit for publication. The corresponding author had full access to the study data and final responsibility for the decision to submit for publication.

Funding SDR was funded by Medical Research Council (MRC)-SCORT. ZCD and JF were funded by UK NEQAS for Molecular Genetics.

Disclaimer SDR and RB have received honoraria from UK NEQAS for Molecular Genetics.

Competing interests SDR, honoraria from UK NEQAS for Molecular Genetics; RB, honoraria from Astra Zeneca, Merck, Pfizer and UK NEQAS for Molecular Genetics; advisory role payments from Astra Zeneca and Merck; additional expenses from Astra Zeneca, Merck and Pfizer; ZCD, honoraria from Astra Zeneca; advisory role payments from Astra Zeneca, Amgen and Pfizer.

Provenance and peer review Not commissioned; externally peer reviewed.

Open Access This is an Open Access article distributed in accordance with the terms of the Creative Commons Attribution (CC BY 4.0) license, which permits others to distribute, remix, adapt and build upon this work, for commercial use, provided the original work is properly cited. See: http://creativecommons.org/licenses/ by/4.0/

\section{REFERENCES}

1 Bokemeyer C, Bondarenko I, Hartmann JT, et al. Efficacy according to biomarker status of cetuximab plus FOLFOX-4 as first-line treatment for metastatic colorectal cancer: the OPUS study. Ann Oncol 2011;22:1535-46.

2 Van Cutsem E, Köhne $\mathrm{CH}$, Hitre $\mathrm{E}$, et al. Cetuximab and chemotherapy as initial treatment for metastatic colorectal cancer. N Engl J Med 2009;360:1408-17.

3 Amado RG, Wolf $M$, Peeters $M$, et al. Wild-type KRAS is required for panitumumab efficacy in patients with metastatic colorectal cancer. J Clin Oncol 2008;26:1626-34.

4 Douillard JY, Siena S, Cassidy J, et al. Randomized, phase III trial of panitumumab with infusional fluorouracil, leucovorin, and oxaliplatin (FOLFOX4) versus FOLFOX4 alone as first-line treatment in patients with previously untreated metastatic colorectal cancer: the PRIME study. J Clin Oncol 2010;28:4697-705.

5 Peeters M, Price TJ, Cervantes A, et al. Randomized phase III study of panitumumab with fluorouracil, leucovorin, and irinotecan (FOLFIRI) compared with FOLFIRI alone as second-line treatment in patients with metastatic colorectal cancer. J Clin Oncol 2010;28:4706-13.

6 Douillard JY, Oliner KS, Siena S, et al. Panitumumab-FOLFOX4 treatment and RAS mutations in colorectal cancer. N Engl J Med 2013;369:1023-34.

7 Van Cutsem E, Lenz HJ, Köhne CH, et al. Fluorouracil, leucovorin, and irinotecan plus cetuximab treatment and RAS mutations in colorectal cancer. J Clin Oncol 2015;33:692-700.

8 Adelstein BA, Dobbins TA, Harris CA, et al. A systematic review and meta-analysis of KRAS status as the determinant of response to anti-EGFR antibodies and the impact of partner chemotherapy in metastatic colorectal cancer. Eur J Cancer 2011;47:1343-54.

9 Dahabreh IJ, Terasawa T, Castaldi PJ, et al. Systematic review: anti-epidermal growth factor receptor treatment effect modification by KRAS mutations in advanced colorectal cancer. Ann Intern Med 2011;154:37-49.

10 Health Quality Ontario. KRAS testing for anti-EGFR therapy in advanced colorectal cancer: an evidence-based and economic analysis. Ont Health Technol Assess Ser 2010;10:1-49.

11 Hoyle M, Crathorne L, Peters J, et al. The clinical effectiveness and cost-effectiveness of cetuximab (mono- or combination chemotherapy), bevacizumab (combination with non-oxaliplatin chemotherapy) and panitumumab (monotherapy) for the treatment of metastatic colorectal cancer after first-line chemotherapy 
(review of technology appraisal No.150 and part review of technology appraisal No. 118): a systematic review and economic model. Health Technol Assess 2013;17:1-237.

12 Ibrahim EM, Zekri JM, Bin Sadiq BM. Cetuximab-based therapy for metastatic colorectal cancer: a meta-analysis of the effect of K-ras mutations. Int I Colorectal Dis 2010;25:713-21.

13 Lin AY, Buckley NS, Lu AT, et al. Effect of KRAS mutational status in advanced colorectal cancer on the outcomes of anti-epidermal growth factor receptor monoclonal antibody therapy: a systematic review and meta-analysis. Clin Colorectal Cancer 2011;10:63-9.

14 Linardou H, Dahabreh IJ, Kanaloupiti D, et al. Assessment of somatic k-RAS mutations as a mechanism associated with resistance to EGFR-targeted agents: a systematic review and meta-analysis of studies in advanced non-small-cell lung cancer and metastatic colorectal cancer. Lancet Oncol 2008;9:962-72.

15 Petrelli F, Barni S, Anti Eaflm: Resectability and outcome with anti-EGFR agents in patients with KRAS wild-type colorectal liver-limited metastases: a meta-analysis. Int J Colorectal Dis 2012;27:997-1004.

16 Petrelli F, Borgonovo K, Cabiddu M, et al. Cetuximab and panitumumab in KRAS wild-type colorectal cancer: a meta-analysis. Int J Colorectal Dis 2011;26:823-33.

17 Qiu LX, Mao C, Zhang J, et al. Predictive and prognostic value of KRAS mutations in metastatic colorectal cancer patients treated with cetuximab: a meta-analysis of 22 studies. Eur J Cancer 2010;6:2781-7.

18 Vale CL, Tierney JF, Fisher D, et al. Does anti-EGFR therapy improve outcome in advanced colorectal cancer? A systematic review and meta-analysis. Cancer Treat Rev 2012;38:618-25
19 Zhang L, Ma L, Zhou Q. Overall and KRAS-specific results of combined cetuximab treatment and chemotherapy for metastatic colorectal cancer: a meta-analysis. Int J Colorectal Dis 2011;26:1025-33.

20 Allegra CJ, Rumble RB, Hamilton SR, et al. Extended RAS gene mutation testing in metastatic colorectal carcinoma to predict response to anti-epidermal growth factor receptor monoclonal antibody therapy: American Society of Clinical Oncology Provisional Clinical Opinion Update 2015. J Clin Oncol 2016;34:179-85.

21 Wong NA, Gonzalez D, Salto-Tellez M, et al. RAS testing of colorectal carcinoma-a guidance document from the Association of Clinical Pathologists Molecular Pathology and Diagnostics Group. J Clin Pathol 2014;67:751-7.

22 Sorich MJ, Wiese MD, Rowland A, et al. Extended RAS mutations and anti-EGFR monoclonal antibody survival benefit in metastatic colorectal cancer: a meta-analysis of randomized, controlled trials. Ann Oncol 2015;26:13-21.

23 Deans ZC, Tull J, Beighton G, et al. Molecular genetics external quality assessment pilot scheme for KRAS analysis in metastatic colorectal cancer. Genet Test Mol Biomarkers 2011;15:777-83.

24 Van Cutsem E, Köhne CH, Láng I, et al. Cetuximab plus irinotecan, fluorouracil, and leucovorin as first-line treatment for metastatic colorectal cancer: updated analysis of overall survival according to tumor KRAS and BRAF mutation status. J Clin Oncol 2011;29:2011-19.

25 Seymour MT, Brown SR, Middleton G, et al. Panitumumab and irinotecan versus irinotecan alone for patients with KRAS wild-type, fluorouracil-resistant advanced colorectal cancer (PICCOLO): a prospectively stratified randomised trial. Lancet Oncol 2013;14:749-59.

26 Bokemeyer $\mathrm{C}$, Köhne $\mathrm{CH}$, Ciardiello F, et al. FOLFOX4 plus cetuximab treatment and RAS mutations in colorectal cancer. Eur J Cancer 2015;51:1243-52. 